\title{
Integrated FDG-PET/CT does not make invasive staging of the intrathoracic lymph nodes in non-small cell lung cancer redundant: a prospective study
}

\author{
K G Tournoy, S Maddens, R Gosselin, G Van Maele, J P van Meerbeeck, A Kelles
}

See end of article for authors' affiliations

Correspondence to:

Dr Kurt G Tournoy,

Department of Respiratory Medicine, Ghent University Hospital, De Pintelaan 185, 9000 Ghent, Belgium; kurt. tournoy@ugent.be

Received 5 October 2006 Accepted 10 January 2007

\begin{abstract}
Background: Staging of non-small cell lung cancer (NSCLC) is important for determining choice of treatment and prognosis. The accuracy of FDG-PET scans for staging of lymph nodes is too low to replace invasive nodal staging. It is unknown whether the accuracy of integrated FDG-PET/CT scanning makes invasive staging redundant.

Methods: In a prospective study, the mediastinal and/or hilar lymph nodes in patients with proven NSCLC were investigated with integrated FDG-PET/CT scanning. Pathological confirmation of all suspect lymph nodes was obtained to calculate the accuracy of the fusion images. In addition, the use of the standardised uptake value (SUV) in the staging of intrathoracic lymph nodes was analysed.

Results: 105 intrathoracic lymph node stations from 52 patients with NSCLC were characterised. The prevalence of malignancy in the lymph nodes was $36 \%$. The sensitivity of the integrated FDG-PET/CT scan to detect malignant lymph nodes was $84 \%$ and its specificity was $85 \%$ (positive likelihood ratio 5.64 , negative likelihood ratio 0.19). SUV $V_{\text {max }}, S U V_{\text {mean }}$ and the $S U V_{\text {max }} / S U V_{\text {liver }}$ ratio were all significantly higher in malignant than in benign lymph nodes. The area under the receiver operating curve did not differ between these three quantitative variables, but the highest accuracy was found with the $S U V_{\max } / S U V_{\text {liver }}$ ratio. At a cut-off value of 1.5 for the $S U V_{\text {max }} / \mathrm{SUV}_{\text {liver }}$ ratio, the sensitivity and specificity to detect malignant lymph node invasion were $82 \%$ and $93 \%$, respectively.

Conclusion: The accuracy of integrated FDG-PET/CT scanning is too low to replace invasive intrathoracic lymph node staging in patients with NSCLC. The visual interpretation of the fusion images of the integrated FDG-PET/CT scan can be replaced by the quantitative variable $S U V_{\text {max }} / S U V_{\text {liver }}$ without loss of accuracy for intrathoracic lymph node staging.
\end{abstract}

S taging non-small lung cancer (NSCLC) is an important part of the diagnostic course in patients with lung cancer since it guides the treatment modalities and predicts survival. ${ }^{1}$ While staging with computed tomography (CT) has an important role in the initial staging by providing excellent anatomical information on the extent of the primary tumour $(\mathrm{T}$ denominator), the CT scan has limited ability to differentiate between benign and malignant lymph nodes ( $\mathrm{N}$ denominator). Whole body positron emission tomography (PET) with 18fluoro-2-deoxy-D-glucose (FDG) has a higher accuracy for detecting intrathoracic lymph node metastasis, ${ }^{2-4}$ and demonstrates occult distant metastasis in approximately $10 \%$ of patients. ${ }^{2}$ According to other reports, CT and FDG-PET perform similarly in mediastinal staging. ${ }^{5}$ Because of an unacceptable rate of false positive and false negative findings, FDG-PET has been shown to be insufficiently accurate to replace invasive lymph node staging on tissue specimens. ${ }^{6-8}$ In addition, the visual localisation of intrathoracic lymph nodes with FDG-PET is not always unequivocal because of the low spatial resolution of the PET images.

Integrated FDG-PET/CT scans theoretically overcome this problem because of the co-acquisition of CT and FDG-PET images resulting in so-called fusion images. Lardinois et al ${ }^{9}$ showed that, in 50 patients with NSCLC, the integrated FDGPET/CT scan was more accurate than FDG-PET alone for nodal staging; however, no difference in accuracy was noted when integrated FDG-PET/CT scans were compared with CT scans alone. Several investigators have indicated that the maximum standardised uptake value $\left(\mathrm{SUV}_{\max }\right)$ of the primary tumour is a prognostic factor in patients with NSCLC. ${ }^{11}{ }^{11}$ With the integrated FDG-PET/CT scan, the application of this quantitative technique at the level of the lymph node becomes more precise since the anatomical borders of the lymph nodes can be exactly identified for determining the region of FDG uptake. ${ }^{12}$

From a clinical point of view, an accuracy or a negative/ positive predictive value of at least $90-95 \%$ for the integrated FDG-PET/CT scan is required to make invasive staging redundant. To evaluate whether tissue-confirmed lymph node staging by surgery or echo-endoscopy can be avoided, we prospectively assessed the accuracy of the integrated FDG-PET/ CT scan in the nodal staging of NSCLC. In addition, we investigated whether an objective measure of FDG uptake based on SUV values could substitute for the subjective interpretation of the fusion images.

\section{METHODS}

\section{Patients}

Consecutive patients with suspected or pathologically proven primary NSCLC were eligible if a tissue specimen from at least one of the intrathoracic lymph nodes was available and if they underwent an integrated FDG-PET/CT scan. All investigations were done before the start of any treatment, and both the integrated PET/CT scan and examination of lymph node tissue

Abbreviations: CT, computed tomography; EBUS-TBNA, endobronchial endoscopic ultrasound with real-time guided transbronchial needle aspiration; EUS-FNA, endoscopic ultrasound with real-time guided fine needle aspiration; FDG, 18-fluoro-2-deoxy-D-glucose; NSCLC, non-small cell lung cancer; PET, positron emission tomography; ROC, receiver operating curve; SUV, standardised uptake value 


\begin{tabular}{|ll|}
\hline \multicolumn{2}{|l|}{ Table 1 Characteristics of patients and } \\
investigations \\
\hline \multicolumn{2}{|c|}{$\mathbf{n}$} \\
\hline Number of patients & 52 \\
Lymph nodes with pathology & 105 \\
Sex & \\
Male & $39(75 \%)$ \\
Female & $13(25 \%)$ \\
Median (range) age (years) & $68(48-80)$ \\
Site of lung tumour & \\
LUL & $9(17 \%)$ \\
LLL & $12(23 \%)$ \\
RUL & $17(33 \%)$ \\
RLL & $12(23 \%)$ \\
RML & $2(4 \%)$ \\
Primary tumour pathology obtained & $35(67 \%)$ \\
With bronchoscopy & $17(49 \%)$ \\
With transthoracic puncture (CT guided) & $5(14 \%)$ \\
With a surgical procedure & $13(37 \%)$ \\
Site of thoracic lymph nodes & $50(48 \%)$ \\
Upper mediastinum (2-3-4-5-6) & $32(31 \%)$ \\
Subcarinal region (7) & $12(11 \%)$ \\
Lower mediastinum (8-9) & $11(10 \%)$ \\
Hilar lymph nodes (10-11) & $50(96 \%)$ \\
Lymph node pathology obtained & $21(42 \%)$ \\
With mediastinoscopy/surgery & $24(48 \%)$ \\
With EUS-FNA or EBUS-TBNA & $5(10 \%)$ \\
With EUS-FNA or EBUS-TBNA followed by & \\
surgical staging & \\
\hline LUL, left upper lower; LL, left lower lobe; RUL, right upper lobe; \\
RLL, right lower lobe; RML, right middle lobe; EUS-FNA, \\
endoscopic ultrasound with real-time guided fine needle \\
aspiration; EBUS-TBNA, endobronchial endoscopic ultrasound \\
with real-time guided transbronchial needle aspiration. \\
\hline
\end{tabular}

were performed within 14 days of each other. The study was approved by the ethics committee of Ghent University Hospital.

\section{Pathology of primary lung tumours and of intrathoracic lymph nodes}

A tissue specimen of the primary tumour was obtained for pathological examination by either bronchoscopy, CT-guided transthoracic puncture or a surgical procedure (thoracotomy or video-assisted thoracoscopy). For intrathoracic lymph nodes, a tissue sample was obtained either by mediastinoscopy, surgical resection or by linear endoscopic ultrasound. The latter consisted of either oesophageal endoscopic ultrasound with real-time guided fine needle aspiration (EUS-FNA) or endobronchial endoscopic ultrasound with real-time guided transbronchial needle aspiration (EBUS-TBNA). Because the negative predictive values of EUS-FNA and EBUS-TBNA are considered too low, ${ }^{13}{ }^{14}$ surgical confirmation was always done in case no malignant lymph node invasion could be demonstrated by either of these endoscopic techniques.

\section{Staging procedures with CT and FDG-PET/CT}

Patients fasted for at least 6 hours, after which blood glucose levels were determined to ascertain a level of $<200 \mathrm{mg} / \mathrm{dl}$. Patients then received $4 \mathrm{MBq} / \mathrm{kg}$ FDG intravenously followed by $250 \mathrm{ml}$ sodium chloride and $20 \mathrm{mg}$ furosemide. For muscle relaxation and to minimise background staining, diazepam $5 \mathrm{mg}$ was given orally. Image acquisition started $60 \mathrm{~min}$ after injection of FDG in a relaxed supine position with the arms alongside the body using an integrated FDG-PET/CT scanner (Philips Gemini FDG-PET/CT, Philips Medical Systems, Cleveland, Ohio, USA). First, a total body low-dose CT scan for calculation of the attenuation correction was performed $(120 \mathrm{kV}$, effective tube current-time product maximum $30 \mathrm{mAS}$, pitch 0.9 , collimation $16 \times 1.5 \mathrm{~mm}$, rotation time $0.5 \mathrm{~s}$, reconstructed contiguous slices of $5 \mathrm{~mm}$, scan field from head up to the upper tights). Second, a scan was performed with a dual head injector (175 mAS, otherwise the same scan parameters) after intravenous injection of $120 \mathrm{ml}$ contrast medium with an iodine concentration of $300 \mathrm{mg} / \mathrm{ml}$ at a flow rate of $1.8 \mathrm{ml} / \mathrm{s}$ followed by a saline flush. No oral contrast was administered. Next, the FDG-PET scan from the orbitomeatal region up to the upper tights (consisting of 8-9 bed positions of 3 min per table position) was performed. Patients were instructed to breathe normally during the acquisition of the CT and FDG-PET/CT images. PET image data sets were reconstructed iteratively using a row action maximum likelihood algorithm with segmented correction for attenuation with use of the CT data. Co-registered images were displayed by means of SYNTEGRA software (Philips Medical Systems).

The CT and integrated FDG-PET/CT scans represented a single procedure of data acquisition but were read separately. For the CT analysis the radiologist was blinded to the FDG-PET data. All intrathoracic lymph nodes were noted and the small and long axes were measured $(\mathrm{mm})$. A lymph node with a short axis of at least $10 \mathrm{~mm}$ was indicated as suspect. The FDG-PET/ CT scan was interpreted based on both CT and FDG-PET images which were read by a nuclear physician and a radiologist.

\section{Determination of FDG-PET/CT SUV variables}

The maximum and mean SUV values were determined by drawing regions of interest on the attenuation-corrected FDGPET fusion images around the primary tumour or the involved lymph node. The variables $S_{U} V_{\max }$ and $S_{U V}$ mean were then calculated as the maximum and mean SUV values, respectively, within the region of interest. Quantitative evaluation based on the $\mathrm{SUV}_{\max } / \mathrm{SUV}_{\text {liver }}$ ratio was calculated as the ratio of the SUV $_{\text {max }}$ over the mean SUV value obtained from the homogenous distribution of radioactivity in the liver. ${ }^{15}$

\section{Statistical analysis}

A clinical research form was completed for each patient and integrated in an electronic database for analysis with SPSS 14.0 (SPSS Inc, Chicago, Illinois, USA). Except for the demographic data, calculations were primarily performed at the single lymph node level. The test characteristics of the CT scan and integrated FDG-PET/CT scan were also calculated at the individual patient level. Values are expressed as mean (SD). Continuous variables were compared with the Mann-Whitney U test and KruskalWallis test. Comparison of proportions (accuracy analysis) was done with a Z-test. A two-sided $p$ value of $<0.05$ was considered statistically significant. The receiver operating curves (ROC) and the ROC areas, the latter being a parameter to measure how well a certain variable can distinguish between benign and malignant lymph nodes, were analysed with SPSS 14.0 and compared with Medcalc Version 9.1.0.1.

\section{RESULTS}

\section{Characteristics of patients and procedures}

The study included 52 patients with NSCLC whose characteristics are shown in table 1. Patients were recruited between November 2005 and April 2006. A diagnosis of NSCLC was obtained in the primary tumour specimen in $67 \%$ of cases. For the others, the diagnosis was extrapolated from the malignant cells found in the lymph node specimen. $52 \%$ of the patients had at least one malignant intrathoracic lymph node. Integrated FDG-PET/CT analysis and tissue specimens were obtained for 105 intrathoracic lymph nodes from 50 patients (an average of 2.01 lymph nodes per patient). In two patients the integrated FDG-PET/CT scan was useful for evaluating the primary tumour only as its central location precluded a confident discrimination of mediastinal lymph nodes. In $10 \%$ of the patients a confirmatory surgical staging technique was 
Table 2 Mean (SD) PET/CT data for the primary tumour in patients with non-small cell lung cancer (NSCLC)

\begin{tabular}{lllll}
\hline & $\mathbf{N}$ & SUV $_{\text {max }}$ & SUV $_{\text {mean }}$ & SUV $_{\text {max }} /$ SUV $_{\text {liver }}$ \\
\hline All NSCLC & 52 & $8.34(4.02)$ & $4.74(2.57)$ & $4.78(2.18)$ \\
Adenocarcinoma & $17(33 \%)$ & $7.50(3.79)$ & $4.72(3.12)$ & $4.06(2.22)$ \\
Squamous cell carcinoma & $20(38 \%)$ & $9.04(4.61)$ & $4.91(2.85)$ & $5.37(2.30)$ \\
Large cell carcinoma & $10(19 \%)$ & $8.32(3.41)$ & $4.46(1.20)$ & $4.38(1.33)$ \\
Adenosquamous carcinoma & $5(10 \%)$ & $8.47(4.08)$ & $4.71(1.82)$ & $5.64(2.51)$ \\
\hline T1 tumours $(\leqslant 3 \mathrm{~cm})$ & $16(31 \%)$ & $6.65(3.42)$ & $3.71(1.87)$ & $3.77(1.52)$ \\
T2-4 tumours $(>3 \mathrm{~cm})$ & $36(69 \%)$ & $9.10(4.09)$ & $5.20(2.73)$ & $5.23(2.29)$ \\
Mann-Whitney U test & & $\mathrm{p}=0.026$ & $\mathrm{p}=0.036$ & $\mathrm{p}=0.008$ \\
\hline SUV, standardised uptake value. & & & \\
\hline
\end{tabular}

necessary because of a negative endoscopic ultrasound examination.

\section{Standardised uptake values for the primary tumour}

Table 2 shows the mean values of the different SUV variables for the primary tumours. There was no difference in $S_{U V}$ max, $S U V_{\text {mean }}$ or in the ratio $S U V_{\max } / S U V_{\text {liver }}$ between the different histological subtypes of NSCLC (Kruskal-Wallis test), nor between squamous and non-squamous carcinomas. Both standard deviation (table 2) and variance (data not shown) of the SUV variables indicate that the spread of the SUV $\max$ was considerably larger than for $\mathrm{SUV}_{\text {mean }}$ or the $\mathrm{SUV}_{\text {max }} / \mathrm{SUV}_{\text {liver }}$ ratio. In addition, lung tumours $<3 \mathrm{~cm}$ had a statistically significant lower $\mathrm{SUV}_{\text {max }}, \mathrm{SUV}_{\text {mean }}$ and $\mathrm{SUV}_{\text {max }} / \mathrm{SUV}_{\text {liver }}$ than tumours $>3 \mathrm{~cm}$. We did not find a significant correlation between the four $\mathrm{T}$ stages and the respective SUV values (data not shown).

\section{Accuracy of CT and integrated FDG-PET/CT for staging lymph nodes}

The accuracy of the CT findings and of the integrated FDG-PET/ CT fusion images for staging intrathoracic lymph nodes is shown in table 3 . The prevalence of malignant lymph nodes was $36 \%$. Whereas the sensitivity of CT and integrated FDG$\mathrm{PET} / \mathrm{CT}$ scans to detect malignant lymph node invasion was equal (both 84\%), integrated FDG-PET/CT scanning had a specificity of $85 \%$ compared with $61 \%$ for the CT scan. The accuracy for detecting malignant intrathoracic lymph nodes was $69 \%$ and $85 \%$, respectively, for CT scans and integrated FDG-PET/CT scans. These figures do not differ significantly from the accuracy of the CT scan (74\%) and integrated FDGPET/CT scan (84\%) calculated at the level of the individual patient. The negative predictive value of small lymph nodes ( short axis $<10 \mathrm{~mm}$ ) without uptake of FDG was 91\% (95\% CI $77 \%$ to $97 \% ; n=43$ ); the positive predictive value of enlarged lymph nodes (short axis >10 mm) with FDG uptake was $79 \%$ ( $95 \%$ CI $62 \%$ to $90 \%$; $\mathrm{n}=38$ ); the negative predictive value of enlarged lymph nodes without uptake of FDG was 90\% (95\% CI $60 \%$ to $98 \% ; n=20$ ); and the positive predictive value of small lymph nodes with FDG uptake was 50\% (95\% CI 6\% to 93\%; $\mathrm{n}=4$ ).

\section{Standardised uptake values for intrathoracic lymph nodes and ROC curves}

Figure 1 shows the SUV-based quantitative integrated FDGPET/CT characteristics for intrathoracic lymph node staging. A statistically significant difference was observed between benign and malignant lymph nodes for $S U V_{\text {max }}, S U V_{\text {mean }}$ and for the $\mathrm{SUV}_{\max } / \mathrm{SUV}_{\text {liver }}$ ratio. This difference was found in the enlarged lymph nodes but not in the smaller ones (data not shown). The ROC curves, ROC area and cut-off values are shown in fig 2. No statistical difference between these three variables was found. For $\mathrm{SUV}_{\max }$ the cut-off value for the highest accuracy was 2.9 , which corresponds to a sensitivity of $76 \%$ and a specificity of $90 \%$. For SUV mean the highest accuracy was reached at a cut-off point of 2.3 , yielding a sensitivity of

Table 3 Accuracy of interpretation of computed tomography (CT) scans versus integrated whole body positron emission tomography with 18-fluoro-2-deoxy-D-glucose/CT (FDG-PET/ CT) fusion images for staging of intrathoracic lymph nodes in patients with non-small cell lung cancer (NSCLC)

\begin{tabular}{llll}
\hline & $\begin{array}{l}\text { All lymph } \\
\text { nodes } \\
\mathbf{n}=105\end{array}$ & $\begin{array}{l}\text { Benign lymph } \\
\text { nodes } \\
\mathbf{n = 6 7}(64 \%)\end{array}$ & $\begin{array}{l}\text { Malignant lymph } \\
\text { nodes } \\
\mathbf{n}=38(36 \%)\end{array}$ \\
\hline CT read-out & $58(55 \%)$ & $26(39 \%)$ & $32(84 \%)$ \\
CT positive (short axis range 10-40 mm) & $47(45 \%)$ & $41(61 \%)$ & $6(16 \%)$ \\
CT negative (short axis range 1-9 mm) & $84(68-93)$ & & \\
CT sensitivity & $61(48-73)$ & & \\
CT specificity & $2.17(1.56-3.02)$ & & \\
CT LR+ & $0.26(0.12-0.55)$ & & \\
CT LR- & $42(40 \%)$ & $10(15 \%)$ & \\
PET/CT read-out & $63(60 \%)$ & $57(85 \%)$ & \\
PET/CT positive (fusion images) & $84(68-93)$ & & \\
PET/CT negative (fusion images) & $85(74-92)$ & & \\
PET/CT sensitivity & $5.64(3.13-10.16)$ & & \\
PET/CT specificity & $0.19(0.09-0.39)$ & & \\
PET/CT LR+ & & & \\
PET/CT LR- & & & \\
\hline
\end{tabular}

LR+, positive likelihood ratio; LR-, negative likelihood ratio.

Statistical analysis CT vs integrated PET/CT read-out: sensitivity, NS; specificity, $p=0.002$; positive likelihood ratio, $\mathrm{p}<0.001$; negative likelihood ratio, NS. 

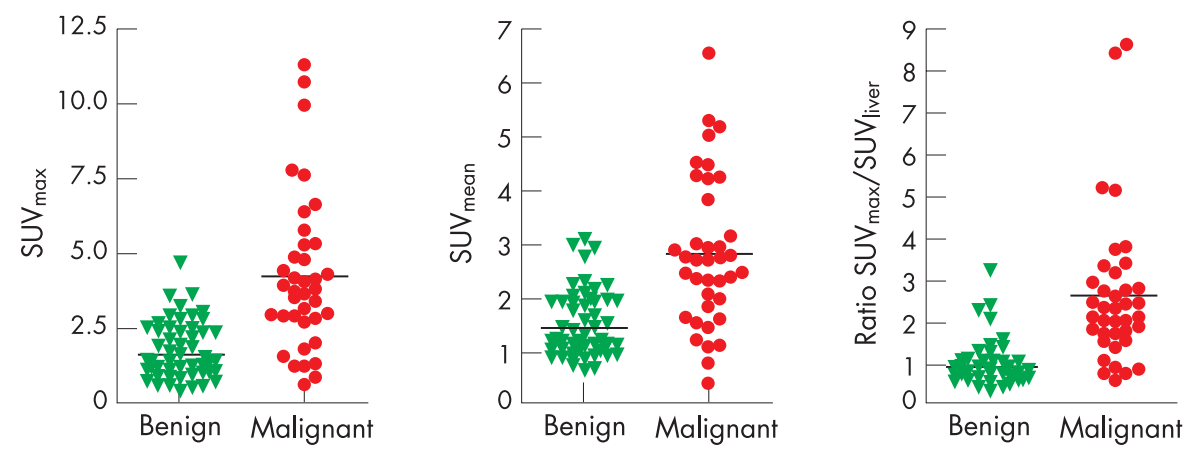

Figure 1 Distribution of the different standardised uptake values (SUV) according to the pathological state of the intrathoracic lymph node (LN).

\begin{tabular}{cccc}
\hline Mean $(S D)$ & $\mathrm{SUV}_{\text {max }}$ & $\mathrm{SUV}_{\text {mean }}$ & $\mathrm{SUV}$ max $/ S U V_{\text {liver }}$ \\
\hline $\begin{array}{c}\text { Benign LNs } \\
\mathrm{N}=67(64 \%)\end{array}$ & $1.69(0.88)$ & $1.39(0.64)$ & $0.94(0.49)$ \\
Malignant $\mathrm{LNs}$ & $4.28(2.59)^{*}$ & $2.84(1.40)^{*}$ & $2.63(1.79)^{*}$ \\
$\mathrm{~N}=38(36 \%)$ & $\mathrm{p}<0.001$ & $\mathrm{p}<0.001$ & $\mathrm{p}<0.001$ \\
Mann-Whitney U-test & &
\end{tabular}

$68 \%$ and a specificity of $93 \%$. The $\mathrm{SUV}_{\max } / \mathrm{SUV}_{\text {liver }}$ ratio had at a cut-off value of 1.5 , the highest accuracy with a sensitivity of $82 \%$ and a specificity of $93 \%$.

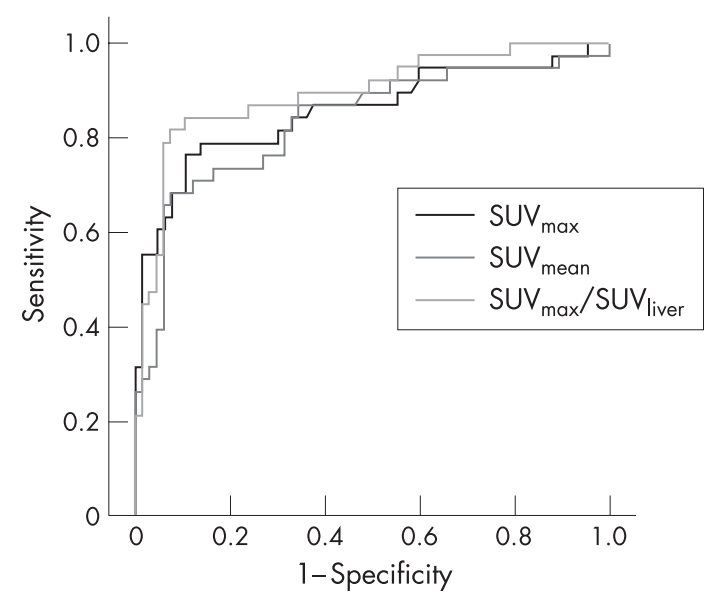

\begin{tabular}{cccc}
\hline & $\mathrm{SUV}_{\text {max }}$ & $\mathrm{SUV}_{\text {mean }}$ & $\mathrm{SUV}_{\text {max }} / \mathrm{SUV}_{\text {liver }}$ \\
\hline ROC area & 0.857 & 0.835 & 0.894 \\
\hline
\end{tabular}

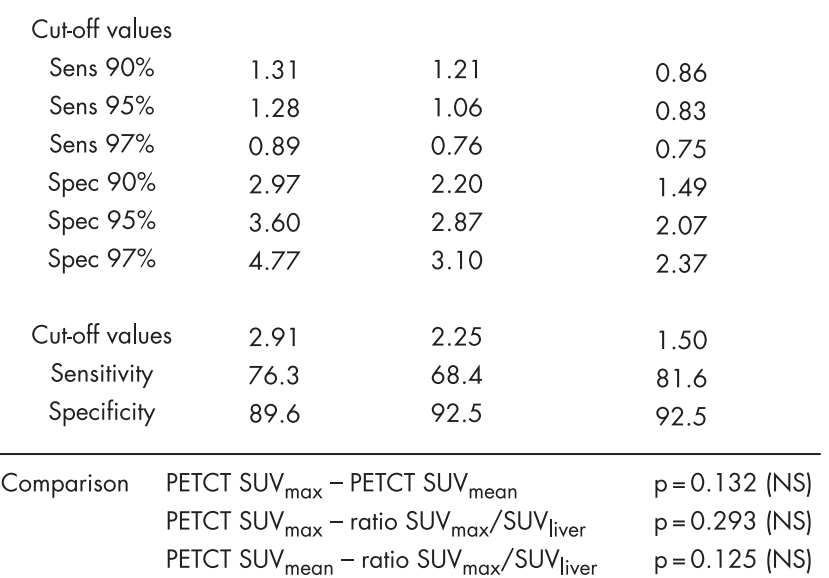

Figure 2 Receiver operating characteristic (ROC) curves for intrathoracic lymph node staging with PET/CT variables. SUV, standardised uptake value.

\section{DISCUSSION}

In this prospective study we have analysed the value of the integrated FDG-PET/CT scan for assessing intrathoracic lymph nodes in patients with NSCLC. Its accuracy for predicting malignant lymph node invasion was found to be higher than with a CT scan. We also found that the $\mathrm{SUV}_{\text {max }} / \mathrm{SUV}_{\text {liver }}$ ratio, a quantitative measure of FDG uptake, predicts malignant invasion in the intrathoracic lymph node with an accuracy comparable to that obtained with the integrated FDG-PET/CT fusion images. However, neither the interpretation of the integrated FDG-PET/CT fusion images nor the calculated cutoffs enabled us to discontinue using tissue-based lymph node staging in NSCLC because all the test characteristics were below the threshold of $95 \%$ at which malignant lymph node invasion can be confidently ruled in or out. Therefore, neither a positive nor a negative integrated FDG-PET/CT scan allows the clinician to confidently predict whether or not an intrathoracic lymph node is malignant.

The advantage of the integrated FDG-PET/CT scan over the CT scan as well as the FDG-PET scan in thoracic oncology is the fact that the spatial resolution of the integrated scan is much higher than with the FDG-PET scan. Because of co-acquisition and fusion, FDG uptake can be exactly localised even in relatively small focal abnormalities such as intrathoracic lymph nodes. This allows a detailed analysis of the value of SUV-based variables within a certain area of interest defined by the anatomical borders. We chose $S_{U V}$ max, $S U V_{\text {mean }}$ and a tumour-to-background ratio $\mathrm{SUV}_{\max } / \mathrm{SUV}_{\text {liver }}$ and applied these parameters not only to primary tumours but also to intrathoracic lymph nodes.

It has been shown in several retrospective series that the SUV $_{\text {max }}$ obtained with FDG-PET scanning of the primary tumour predicts both stage and survival of the patient with NSCLC. ${ }^{1011}$ A correlation between $\mathrm{T}$ stage and SUV $\max$ was found by Cerfolio et $\mathrm{al}^{11}$ for $\mathrm{T} 1$ and $\mathrm{T} 2$ tumours but not for T3 and T4 tumours. The same authors also suggested that squamous cell carcinomas have a higher $S_{U V} V_{\max }$ than other pathological subtypes. ${ }^{11} \mathrm{SUV}_{\max }$ was proposed as a variable because it was suggested that its reproducibility is better than for SUV mean. ${ }^{16}$ However, others have suggested that $\mathrm{SUV}_{\text {mean }}$ as well as the $\mathrm{SUV}_{\text {max }} / \mathrm{SUV}_{\text {liver }}$ ratio give more stable and reliable results. ${ }^{15}$

In our prospective series we analysed these SUV-based characteristics of the primary tumour with integrated 
FDG-PET/CT scanning and found that the clustering of $S U V_{\text {mean }}$ and the $S U V_{\text {max }} / S_{U V}$ liver ratio was better than that

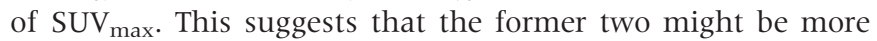
reliable and stable than $S_{U V}$ max. Although our data also confirm that $\mathrm{Tl}$ tumours had a lower $\mathrm{SUV}_{\max }$ than T2-4 tumours, ${ }^{11}$ we did not observe a difference between the different pathological subtypes.

For lymph node staging, we found that the integrated FDGPET/CT scan performed better than the CT scan in terms of specificity ( $85 \%$ vs $61 \%, p=0.002$ ) but not sensitivity $(84 \%$ for both). It is already known that FDG-PET scans are better than CT scans for staging of intrathoracic lymph nodes. ${ }^{6-8} 17$ However, Shim et al ${ }^{18}$ directly compared the accuracy of CT and integrated FDG-PET/CT scanning and suggested that the integrated FDG-PET/CT scan is superior for lymph node staging. Our data confirm this finding and further suggest that a visual interpretation of the fusion images is not sufficiently accurate to avoid invasive tissue specimen-based lymph node staging. Because the implication of a false positive staging evaluation is a missed opportunity for surgical care, we confirm that, as for FDG-PET, a positive integrated FDG-PET/CT result does not confidently rule in lymph node invasion. de Langen et a ${ }^{19}$ found that lymph nodes measuring 10-15 mm without FDG uptake had a post-test probability for malignant invasion of only $5 \%$, so they suggested that these patients do not need further mediastinal investigation. Since the absolute number of false negative lymph nodes in our series was low, we are cautious about drawing firm conclusions on the negative predictive value of integrated FDG-PET/CT scans. Nevertheless, we calculated a specificity of $85 \%$ and a negative predictive value of $90 \%$, which compares with the findings of Shim et al. ${ }^{18}$

We also determined whether the accuracy of intrathoracic lymph node staging could be improved by calculating SUV variables on the fusion images. As expected, we found that enlarged malignant lymph nodes have significantly higher SUV values than benign ones. With an FDG-PET scan it was proposed that a SUV max $_{\text {mat }}$ cutf of 4.4 was the best threshold to discriminate between benign and malignant lymph nodes. ${ }^{17}$ With the integrated FDG-PET/CT scan, we calculated ROCs for $\mathrm{SUV}_{\max }$ and also for $\mathrm{SUV}_{\text {mean }}$ and the $\mathrm{SUV}_{\max } / \mathrm{SUV}_{\text {liver }}$ ratio for lymph node staging and found that optimal cut-off points for all three variables could be identified. For $S U_{\max }$ we found that the optimal threshold for discriminating malignancy was 2.9. The discrepancy between published data ${ }^{17}$ and our findings could be partly attributed to the fact that FDG-PET techniques have evolved over the past 10 years resulting in more sensitive cameras, and also to the fact that different technology was used for the attenuation correction and for the calculation of the SUV data. For an FDG-PET examination, an external cesium source is used for calculating the attenuation correction while, for an FDG-PET/CT camera, the CT data are used to calculate the attenuation correction. This is the reason why we also included a ratio as variable $\left(\mathrm{SUV}_{\max } / \mathrm{SUV}_{\text {liver }}\right)$ so that cameraspecific variables could be omitted. In addition, the lower SUV $_{\text {max }}$ threshold in our study could also be related to the fact that we were able to delineate exactly the nodal focus on the integrated FDG-PET/CT fusion images.

More importantly, our results indicate that a thoracic oncology clinic working with FDG-PET or with integrated FDG-PET/CT should not automatically rely on published SUVbased cut-off values but should determine their own machinespecific cut-off points. Although at a cut-off point of 1.5 the accuracy of the $S U V_{\text {max }} / \mathrm{SUV}_{\text {liver }}$ ratio was comparable to the one obtained with the fusion images, calculated SUV values also do not replace lymph node tissue diagnosis.

This study has a number of limitations. A number of methods encompassing echo-endoscopy and surgical staging procedures were used to obtain lymph node pathology. Although all negative echo-endoscopies were confirmed with surgery, it is clear that patients who had proven N2/N3 disease after echoendoscopy did not have full pathological mapping of the mediastinum. In addition, although over 100 lymph nodes were characterised, it is inevitable that-for the assessment of test variables-false negative and false positive findings are calculated on relatively small numbers. As discussed above, this means that the interpretation of negative predictive values of a small lymph node which is FDG-PET/CT negative should be done with caution. Furthermore, the data were not corrected for possible intrasubject correlation between nodes. Although re-calculation of the test characteristics at the patient level did not show a difference from the data obtained at the single lymph node level, this must be taken into account when interpreting the data.

In conclusion, integrated FDG-PET/CT scanning has an overall accuracy which is too low to replace invasive intrathoracic lymph node staging in patients with NSCLC. The visual interpretation of the fusion images of the integrated FDG-PET/ CT scan can be replaced by the quantitative variable $\mathrm{SUV}_{\max } /$ SUV $_{\text {liver }}$ without loss of accuracy for staging of intrathoracic lymph nodes.

\section{ACKNOWLEDGEMENTS}

This is an investigator initiated study.

\section{Authors' affiliations}

K G Tournoy, S Maddens, J P van Meerbeeck, Department of Respiratory Medicine, Ghent University Hospital, Ghent, Belgium

R Gosselin, Department of Radiology, Ghent University Hospital, Ghent, Belgium

G Van Maele, Department of Medical Statistics, Ghent University Hospital, Ghent, Belgium

A Kelles, Department of Nuclear Medicine, Ghent University Hospital, Ghent, Belgium

Funding: None.

Competing interests: None.

\section{REFERENCES}

1 Spira A, Ettinger DS. Multidisciplinary management of lung cancer. N Engl J Med 2004;350:379-92.

2 Pieterman RM, van Putten JW, Meuzelaar JJ, et al. Preoperative staging of nonsmall-cell lung cancer with positron-emission tomography. N Engl J Med 2000;343:254-61.

3 Kalff V, Hicks RJ, MacManus MP, et al. Clinical impact of (18)F fluorodeoxyglucose positron emission tomography in patients with non-small-cell lung cancer: a prospective study. J Clin Oncol 2001;19:111-8.

4 Gould MK, Kuschner WG, Rydzak CE, et al. Test performance of positron emission tomography and computed tomography for mediastinal staging in patients with non-small-cell lung cancer: a meta-analysis. Ann Intern Med 2003;139:879-92.

5 Pozo-Rodriguez F, Martin de Nicolas JL, Sanchez-Nistal MA, et al. Accuracy of helical computed tomography and [18F] fluorodeoxyglucose positron emission tomography for identifying lymph node mediastinal metastases in potentially resectable non-small-cell lung cancer. J Clin Oncol 2005;23:8248-56.

6 Cerfolio RJ, Ojha B, Bryant AS, et al. The role of FDG-PET scan in staging patients with nonsmall cell carcinoma. Ann Thorac Surg 2003;76:861-6.

7 Vansteenkiste JF, Stroobants SG, De Leyn PR, et al. Potential use of FDG-PET scan after induction chemotherapy in surgically staged Illa-N2 non-small-cell lung cancer: a prospective pilot study. The Leuven Lung Cancer Group. Ann Oncol 1998;9:1193-8.

8 Gonzalez-Stawinski GV, Lemaire A, Merchant F, et al. A comparative analysis of positron emission tomography and mediastinoscopy in staging non-small cell lung cancer. J Thorac Cardiovasc Surg 2003;126:1900-5.

9 Lardinois D, Weder W, Hany TF, et al. Staging of non-small-cell lung cancer with integrated positron-emission tomography and computed tomography. N Engl J Med 2003;348:2500-7.

10 Vansteenkiste JF, Stroobants SG, Dupont PJ, et al. Prognostic importance of the standardized uptake value on (18)F-fluoro-2-deoxy-glucose-positron emission tomography scan in non-small-cell lung cancer: an analysis of 125 cases. Leuven Lung Cancer Group. J Clin Oncol 1999;17:3201-6. 
11 Cerfolio RJ, Bryant AS, Ohja B, et al. The maximum standardized uptake values on positron emission tomography of a non-small cell lung cancer predict stage, recurrence, and survival. J Thorac Cardiovasc Surg 2005;130:151-9.

12 De Wever W, Ceyssens S, Mortelmans L, et al. Additional value of PET-CT in the staging of lung cancer: comparison with CT alone, PET alone and visual correlation of PET and CT. Eur Radiol 2007;17:467-73.

13 Kramer H, Groen HJ. Current concepts in the mediastinal lymph node staging of nonsmall cell lung cancer. Ann Surg 2003;238:180-8.

14 Toloza EM, Harpole L, Detterbeck F, et al. Invasive staging of non-small cell lung cancer: a review of the current evidence. Chest 2003;123(1 Suppl):157-66S.

15 Paquet N, Albert A, Foidart J, et al. Within-patient variability of (18)F-FDG: standardized uptake values in normal tissues. J Nucl Med 2004;45:784-8.
16 Lee JR, Madsen MT, Bushnel D, et al. A threshold method to improve standardized uptake value reproducibility. Nucl Med Commun 2000;21:685-90.

17 Vansteenkiste JF, Stroobants SG, De Leyn PR, et al. Lymph node staging in nonsmall-cell lung cancer with FDG-PET scan: a prospective study on 690 lymph node stations from 68 patients. J Clin Oncol 1998;16:2142-9.

18 Shim SS, Lee KS, Kim BT, et al. Non-small cell lung cancer: prospective comparison of integrated FDG PET/CT and CT alone for preoperative staging Radiology 2005;236:1011-9.

19 de Langen AJ, Raijmakers P, Riphagen I, et al. The size of mediastinal lymph nodes and its relation with metastatic involvement: a meta-analysis. Eur J Cardiothorac Surg 2006;29:26-9.

\section{LUNG ALERT}

\section{COPD and Ml: a bad combination}

$\Delta$ Salisbury AC, Reid KJ, Spertus JA. Impact of chronic obstructive pulmonary disease on post-myocardial infarction outcomes. Am J Cardiol 2007;99:636-41.

n this observational prospective multicentre study that enrolled 2481 patients, the authors tried to highlight the relationship between chronic obstructive pulmonary disease (COPD) and a number of outcomes following an acute myocardial infarction (MI).

One-year mortality and rehospitalisation rates were significantly higher in patients with COPD than without (15.8\% vs $5.7 \%$ ) and (48.7\% vs 38.6\%), respectively. In addition, patients with COPD had worse health status at baseline as well as at 1 year and a trend toward a higher prevalence of angina at 1 year. Not surprisingly, patients with COPD had fewer coronary revascularisation procedures and less use of $\beta$ blockers, aspirin and cardiac rehabilitation.

With the well-documented role of $\beta$ blockers in improving survival post-MI even in patients with COPD, it is possible that more frequent usage of these agents, particularly the cardioselective agents, in the study population could have led to a better 1 year survival than observed and possibly a lower prevalence of angina, again at 1 year.

The study used clinical rather than pulmonary function test definition of COPD and as a consequence could have misclassified some patients with possible inclusion of non-COPD patients, for example those with asthma. Furthermore, the outcomes could have been influenced by the severity of COPD, which the authors were unable to classify. Nevertheless, the data suggest that patients with acute MI and COPD have a substantially worse prognosis in terms of mortality and health status. This cohort of patients therefore warrants careful attention and closer follow-up.

Nabil M Al Lawati

Clinical Fellow, University of British Columbia, Canada; drnabilm@yahoo.com 